\title{
Short-Range Atmospheric Dispersion of Carbon Dioxide
}

\author{
Andrea Cortis · Curtis M. Oldenburg
}

Received: 4 February 2009 / Accepted: 6 August 2009 / Published online: 20 August 2009

(C) The Author(s) 2009. This article is published with open access at Springerlink.com

\begin{abstract}
We present a numerical study aimed at quantifying the effects of concentrationdependent density on the spread of a seeping plume of $\mathrm{CO}_{2}$ into the atmosphere such as could arise from a leaking geologic carbon sequestration site. Results of numerical models can be used to supplement field monitoring estimates of $\mathrm{CO}_{2}$ seepage flux by modelling transport and dispersion between the source emission and concentration-measurement points. We focus on modelling $\mathrm{CO}_{2}$ seepage dispersion over relatively short distances where density effects are likely to be important. We model dense gas dispersion using the steady-state Reynoldsaveraged Navier-Stokes equations with density dependence in the gravity term. Results for a two-dimensional system show that a density dependence emerges at higher fluxes than prior estimates. A universal scaling relation is derived that allows estimation of the flux from concentrations measured downwind and vice versa.
\end{abstract}

Keywords Dense-gas dispersion - Geologic carbon sequestration · Geologic $\mathrm{CO}_{2}$ storage . Modelling $\cdot$ Monitoring

\section{Introduction}

Carbon capture and storage (CCS) has emerged as a technology for reducing $\mathrm{CO}_{2}$ emissions into the atmosphere from point sources such as power plants, cement plants, and oil refineries. In CCS, captured $\mathrm{CO}_{2}$ is stored in deep geologic formations (such as depleted oil and gas reservoirs, and brine formations) known as geologic carbon sequestration (GCS) sites. Possible seepage (escape of $\mathrm{CO}_{2}$ from the GCS site into the atmosphere) represents a significant concern from the point of view of safety and effectiveness. In order to ensure the safety and effectiveness of GCS sites, surface monitoring networks to detect, locate, and

\footnotetext{
A. Cortis $(\bowtie) \cdot$ C. M. Oldenburg

Earth Sciences Division, Lawrence Berkeley National Laboratory, One Cyclotron rd., Berkeley, CA, USA e-mail: acortis@lbl.gov

C. M. Oldenburg

e-mail: cmoldenburg@lbl.gov
} 
quantify seepage, if any, are needed (Benson 2006; Cortis et al. 2008). Understanding the dispersion processes occurring above ground between the emission area and surface monitoring instruments is essential to interpreting measurements.

Different measurement methods for $\mathrm{CO}_{2}$ concentration and soil fluxes exist (Oldenburg et al. 2003; Lewicki etal. 2007; Leuning etal. 2008). In order to be detectable, however, the allowed seepage flux should be larger than the average respiration flux by an amplification factor $\lambda$, a measure of the precision with which seepage flux can be distinguished from the background respiration flux (Cortis et al. 2008). According to the optimal strategies introduced by Cortis et al. (2008), monitoring costs directly depend on the value of the amplification factor; enhancing the interpretation and reliability of the measurement methods can thus significantly improve the economics of CCS projects.

While the reliability in $\mathrm{CO}_{2}$ flux and concentration measurements depends critically on the availability of well-designed and controlled field experiments, numerical models can provide an alternative way for increasing our confidence in measured flux values. Numerical atmospheric dispersion models can significantly enhance the reliability of the various measurement methods by providing, (1) predictions of plume profiles over time and space, and (2) indications of measurement errors due to meteorological parameter sensitivity. Following the tragedies of the natural degassing of the Cameroonian Lake Monoun in 1984 and Lake Nyos in 1986, and the industrial accidents in Seveso, Italy in 1976 and Bophal, India in 1984, intense research efforts have been directed towards understanding the physics of large denser-than-air toxic plumes over significantly large distances. Thorough literature reviews can be found in the classical works of Pasquill and Smith (1983), Britter and McQuaid (1988), and Arya (1999), which describe models of the evolution of dense-gas plume on distances ranging from a few hundred metres to a few kilometres.

When considering static monitoring networks for seepage detection at GCS sites, the typical spacing between eddy-covariance towers (ECT) is estimated to be well below the kilometre scale (Cortis etal. 2008), and short-distance models for the evolution of the $\mathrm{CO}_{2}$ plumes need thus to be considered. Despite the occurrence of small-scale accidents such as at the Idaho National Engineering and Environmental Laboratory (INEEL 1999) where a total flood-type $\mathrm{CO}_{2}$ fire extinguishing system discharged without warning into a room resulting in a fatality and injuries, the short-distance evolution of dense-gas plumes has received less attention in the scientific literature. Exceptions include the extensive work of Hanna and Steinberg (2001), Hanna and Chang (2001), and Snyder (2001). A comparison of six widely used dense-gas dispersion models can be found in Hanna et al. (2008).

Extrapolation of long-distance models to short distances is often not justified, and experimental data are lacking. A coupled subsurface-atmospheric flux model was presented by Oldenburg and Unger (2004), but did not take into account the effects of variable density just above the ground in the atmospheric boundary layer. Macedonio and Costa (2002) proposed a model that takes into account the effects of density and seeping gas temperature. Costa et al. (2005) proposed a model where only the concentration equation coupled with the wind mass conservation is solved, and Costa et al. (2008) proposed that a shallow layer approach can be used for the density-dominated regime.

The aim of this work is to provide generalised results of concentration profiles of dense-gas plumes that may be used to improve the degree of confidence that we have in field atmospheric measurements. To this end, we present a series of numerical simulations aimed at modelling the short-distance concentration profiles of dense-gas plumes subject to atmospheric dispersion. The approach is based on the coupling of incompressible, isothermal, steady-state Navier-Stokes (NS) equations for the flow, where air density and viscosity depend locally on the value of the $\mathrm{CO}_{2}$ concentration. In particular the effects of density on flow and transport 
are examined and both quantitative and qualitative features of this type of transport are identified, which provide guidelines and numerical tools to expedite evaluation of the quantity of interest even for a real-time, field set-up.

\section{Physical Model}

For steady-state subsonic isothermal flows, compressible fluids (such as air at standard conditions) may be approximated as incompressible fluids, where the governing NS equations can be written as:

$$
\begin{gathered}
\nabla \cdot \mathbf{v}=0, \\
\rho(\mathbf{v} \cdot \nabla) \mathbf{v}+\nabla p-\left[\nabla \eta\left(\nabla \mathbf{v}+(\nabla \mathbf{v})^{T}\right)\right]=\mathbf{f},
\end{gathered}
$$

where $p$ is pressure $(\mathrm{Pa}), \mathbf{v}\left(\mathrm{ms}^{-1}\right)$ is the fluid velocity, $\rho$ is the density $\left(\mathrm{kg} \mathrm{m}^{-3}\right), \eta$ is the dynamic viscosity (Pas), and $\mathbf{f}=-\rho g \mathbf{e}_{z}$ represents the gravitational force (per unit volume) $\left(\mathrm{N} \mathrm{m}^{-3}\right)$ in the downwards vertical direction. Under the incompressibility assumption, density $\rho$ is time independent and mass conservation is represented by (1a). Real-world atmospheric flow conditions are characterised by the time dependence of the boundary conditions and especially by their inherent three-dimensional character. While these factors play a significant role in dense-gas dispersion models, in this work we focus only on two-dimensional steady-state flow conditions. Atmospheric boundary-layer models typically introduce the so-called Boussinesq assumption in which the variation of density is neglected except in the gravitational force term $\mathbf{f}$. In the context of this approximation, the flows induced by density variations in the inertial terms, $\rho(\mathbf{v} \cdot \nabla) \mathbf{v}$, are considered negligible, a reasonable assumption for flows characterised by small density gradients. For relatively large $\mathrm{CO}_{2}$ fluxes and in particular at the edge of the plume, the validity of the Boussinesq assumption may be questionable. For this reason, we do not assume a Boussinesq approximation for the inertial terms, i.e., the density factor in the inertial terms is not a fixed constant value, but rather is a function of the spatially-varying $\mathrm{CO}_{2}$ mass fraction, $\rho=\rho(x, y, z)$. Atmospheric flows are generally turbulent, i.e., they are characterised by large Reynolds numbers, $R e=\rho v L / \eta$, where $L$ is a characteristic length scale of the fully developed turbulence. As NS equations admit analytical solutions only for the simplest geometries, numerical methods of solution are needed. Two main numerical solution strategies can be identified: (i) direct numerical simulations (DNS) and (ii) turbulence closure models. In DNS the entire range of spatial and temporal scales of turbulence must be resolved in the computational mesh, from the smallest dissipative scale, up to the integral scale $L$. The smallest dissipative scale that needs to be considered is the so-called Kolmogorov scale $\ell=\left(v^{3} / \varepsilon^{1 / 4}\right)$, where $\varepsilon \simeq v^{\prime 3} / L$ is the rate of kinetic energy dissipation, where $v^{\prime}$ are velocity fluctuations. With these constraints, the memory required for a DNS can be estimated on the order of $R e^{9 / 4}$, whereas the number of operations grows as $R e^{3}$, and the use of supercomputers becomes necessary (Tu et al. 2008).

Turbulence models represent an alternative approach, in which turbulence effects are modelled on top of a spatially-averaged version of the NS equations. The key observation for this type of modelling is that at large Reynolds numbers eddy-flow structures appear at all scales of observation, down to the Kolmogorov scale where energy is dissipated into heat by viscous forces. Generally, however, when examining dispersion we are interested in averages over length and time scales much larger than almost all the eddies and all the relevant quantities can be conveniently decomposed as the sum of their mean value and a perturbation, e.g., $\mathbf{v}=\overline{\mathbf{v}}+\mathbf{v}^{\prime}$. Substitution into the NS equations of these decompositions for velocity and 
pressure, yields the so-called Reynolds-averaged NS equations

$$
\begin{gathered}
\nabla \cdot \overline{\mathbf{v}}=0, \\
\rho(\overline{\mathbf{v}} \cdot \nabla) \overline{\mathbf{v}}+\nabla \bar{p}-\left[\nabla \eta\left(\nabla \overline{\mathbf{v}}+(\nabla \overline{\mathbf{v}})^{T}\right)+\nabla\left(\rho \overline{\mathbf{v}^{\prime} \mathbf{v}^{\prime}}\right)\right]=\mathbf{f} .
\end{gathered}
$$

The Reynolds-averaged NS equations are structurally similar to the NS equations except for the presence of the additional stress term $\nabla\left(\overline{\rho \mathbf{v}^{\prime} \mathbf{v}^{\prime}}\right)$. This additional stress, often called the Reynolds stress, stems from the covariance of the fluctuating velocity field $\mathbf{v}^{\prime}$, the effects of which can be modelled as a macroscopic turbulent viscosity, $\eta_{T}$,

$$
\rho \overline{\overline{\mathbf{v}}^{\prime} \mathbf{v}^{\prime}}-\frac{\rho}{3} \operatorname{tr}\left(\overline{\mathbf{v}^{\prime} \mathbf{v}^{\prime}}\right) \mid=\eta_{T}(\nabla \overline{\mathbf{v}}+(\nabla \overline{\mathbf{v}}))^{T},
$$

where tr indicates the trace operator. The introduction of the turbulent viscosity leads thus to the following form of the Reynolds-averaged NS equations:

$$
\begin{gathered}
\nabla \cdot \overline{\mathbf{v}}=0, \\
\rho(\overline{\mathbf{v}} \cdot \nabla) \overline{\mathbf{v}}+\nabla \bar{p}-\left[\nabla\left(\eta+\eta_{T}\right)(\nabla \overline{\mathbf{v}}+(\nabla \overline{\mathbf{v}}))^{T}\right]=\mathbf{f},
\end{gathered}
$$

where the term $\frac{\rho}{3} \operatorname{tr}\left(\overline{\mathbf{v}^{\prime} \mathbf{v}^{\prime}}\right) \mid$ has been added to the mean pressure $\bar{p}$. The problem is now shifted to finding an estimate of $\eta_{T}$, and for this we need additional closure equations. In this work, we make use of the so-called $k-\varepsilon$ model, which requires the introduction of a set of two coupled partial differential equations for the kinetic energy $k=\frac{1}{2} \overline{v_{i} v_{i}}$, and the dissipation rate of turbulent energy $\varepsilon$ (Stull 1988):

$$
\begin{aligned}
& \rho \frac{\partial k}{\partial t}-\nabla \cdot\left[\left(\eta+\rho \frac{C_{\eta}}{\sigma_{k}} \frac{k^{2}}{\varepsilon}\right) \nabla k\right]+\rho \overline{\mathbf{v}} \cdot \nabla k=\rho C_{\eta} \frac{k^{2}}{2 \varepsilon}\left(\nabla \overline{\mathbf{v}}+(\nabla \overline{\mathbf{v}})^{T}\right)^{2}-\rho \varepsilon, \\
& \rho \frac{\partial \varepsilon}{\partial t}-\nabla \cdot\left[\left(\eta+\rho \frac{C_{\eta}}{\sigma_{\varepsilon}} \frac{k^{2}}{\varepsilon}\right) \nabla \varepsilon\right]+\rho \overline{\mathbf{v}} \cdot \nabla \varepsilon=\rho C_{\varepsilon 1} \frac{k}{2}\left(\nabla \overline{\mathbf{v}}+(\nabla \overline{\mathbf{v}})^{T}\right)^{2}-\rho C_{\varepsilon 1} \frac{\varepsilon^{2}}{k},
\end{aligned}
$$

where $C_{\eta}=0.09, C_{\varepsilon 1}=1.44, C_{\varepsilon 2}=1.92, \sigma_{k}=0.9$, and $\sigma_{\varepsilon}=1.3$ are empirical constants determined from experimental data. The turbulent viscosity is then modelled as $\eta_{T}=\rho C_{\eta} \frac{k^{2}}{\varepsilon}$ (Stull 1988). Boundary conditions for $k$ and $\varepsilon$ must be specified to complete the description of the physical model. This is often difficult and a source of uncertainty since the incoming turbulence is rarely known exactly, and an educated guess of the incoming turbulence is required. Equations (4) together with (5) represent a complete model of the turbulent flow.

In this work, however, our interest rests mainly on the density effects of a dense-gas plume on the flow, and the subsequent plume dispersion. When the concentration of $\mathrm{CO}_{2}$ becomes sufficiently large, the variations of the density and viscosity with respect to the standard atmospheric composition become significant and affect the overall characteristics of the flow. We propose a model where the equations of state for density and viscosity vary locally as a function of the $\mathrm{CO}_{2}$ mass fraction. Density is defined by means of an ideal-mixture mixing rule

$$
\rho=\frac{1}{\frac{X}{\rho_{c}}+\frac{(1-X)}{\rho_{a}}},
$$

where $\rho_{a}=1.18 \mathrm{~kg} \mathrm{~m}^{-3}$ and $\rho_{c}=1.82 \mathrm{~kg} \mathrm{~m}^{-3}$ are the air density and pure $\mathrm{CO}_{2}$ density, respectively and $X$ is the $\mathrm{CO}_{2}$ mass fraction. Air viscosity was tabulated as a function of $\mathrm{CO}_{2}$ mass fraction using the equation of state due to Peng and Robinson (1976) with parameters 
defined in Walas (1985), as implemented in Reagan (2008) and Moridis et al. (2008). The limit values for the viscosity are $\eta_{a}=1.807 \times 10^{-5} \mathrm{Pas}$ and $\eta_{c}=1.461 \times 10^{-5} \mathrm{Pas}$. Both density and viscosity have been considered at constant temperature $T=295 \mathrm{~K}$. At steadystate conditions, the $\mathrm{CO}_{2}$ concentration spatial distribution $c=c(x, y, z)$ induces spatially varying density $\rho=\rho(x, y, z)$ and viscosity $\eta=\eta(x, y, z)$ fields that are accounted for in the incompressible NS equations.

Atmospheric dispersion is often treated by means of a Fickian hypothesis on the flux of the contaminant, i.e., by modelling the concentration flux of the contaminant as proportional to the gradient of the concentration where the proportionality constant is the molecular diffusivity. This is a well-known representation of transport that leads to the classical advection-dispersion equation (ADE)

$$
\frac{\partial c}{\partial t}=-\mathbf{v} \cdot \nabla c+\nabla(D \nabla c)
$$

According to the ADE description in Eq. 7, the trace gas is advected along the flow streamlines and further dispersed across neighbouring streamlines by Brownian motion effects. While the ADE with molecular diffusion can be seen as an appropriate way to describe transport at extremely small scales and more or less homogeneous velocity fields, doubts emerge about the appropriateness of its use at larger scales, where the effects of the turbulence at all scales accumulate. In this work we consider only the steady-state picture of transport, hence $\frac{\partial c}{\partial t}=0$, and will assume that the $\mathrm{CO}_{2}$ background (being uniformly distributed in the computational domain) plays a secondary role in the dispersion process of the seeping dense-gas plume.

The ADE picture of transport has undergone much scrutiny when contaminant transport in heterogeneous geological media is considered. For this specific application, it is now recognised that even small heterogeneity in the flow path can significantly affect macroscopic transport properties giving rise to so-called macro (scale-dependent) dispersion coefficients. This picture presents striking analogies to the case of atmospheric turbulence, where energy is transferred from larger to smaller eddies, leading ultimately to dissipation by viscosity. In other words, a contaminant transported in the atmosphere experiences a whole host of different fluctuating velocities $\mathbf{v}^{\prime}$ that accumulate into apparent macroscopic scale-dependent dispersion coefficients (see, e.g., Berkowitz et al. (2006) and references therein). This explains the difference in the order of magnitude between the molecular diffusion coefficient, which is on the order of $10^{-5} \mathrm{~m}^{2} \mathrm{~s}^{-1}$, and dispersion coefficients fitted to field experimental data, which are reported to be five to six orders of magnitude larger. A regrettable paucity in well-constrained experimental data and solid theoretical explanations for the macroscopic transport behaviour of a contaminant in the atmosphere at $10^{2} \mathrm{~m}$ scale forces us to adopt a constitutive law for the dispersion coefficient that reflects both the details of the computations and is consistent with apparent dispersion measurements (see, e.g., Davis et al. 1986). Specifically we assume that the dispersion coefficient in the ADE is locally (i.e., for each point in space) equal to $D=f \eta_{T} / \rho$. In order to obtain values of $D$ consistent with observed values of the apparent dispersion coefficient, which have been reported to be on the order of $1-5 \mathrm{~m}^{2} \mathrm{~s}^{-1}$, we rescaled the average value of $\eta_{T}$ by means of the factor $f$. The simulations presented in this work, for all flow conditions, use a constant value of $f=100$. While a number of other physical (e.g., soil and air temperature, and moisture) and geographical factors (e.g., soil topography and vegetation) may significantly affect the evolution of the dense-gas plume, we reserve the analysis of these factors to future studies. 


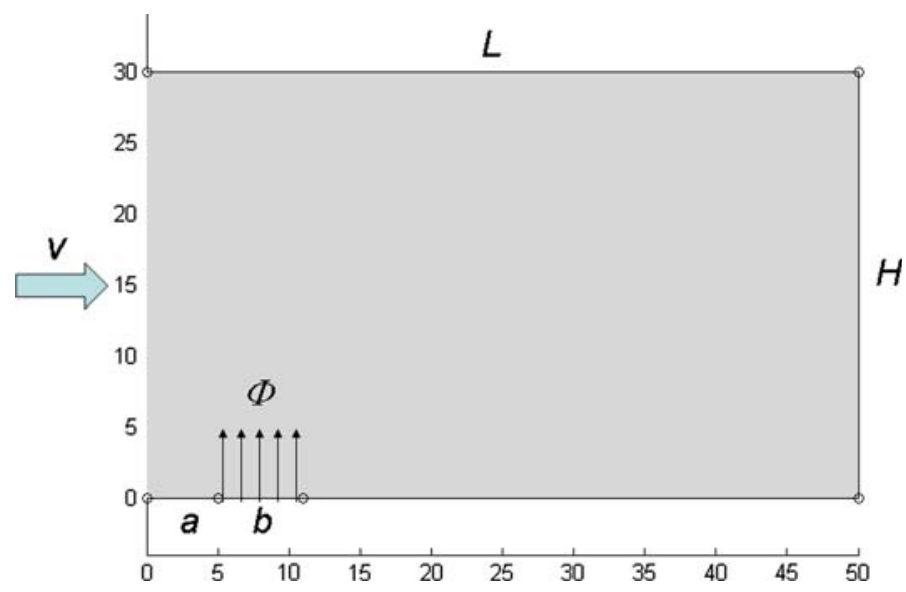

Fig. 1 Sketch of the 2D computational domain (units of $\mathrm{m}$ ). Wind direction is from left to right. A seepage $\mathrm{CO}_{2}$ flux, $\Phi$, is imposed over region of width $b$ at the bottom

\section{Numerical Model}

The coupled system of equations defined by the Reynolds-averaged NS Eq. 2, the $k-\varepsilon$ closure Eq. (5), the advection-dispersion Eq. (7), augmented by the constitutive equations for $\rho=\rho(c), \eta=\eta(c)$, the phenomenological expression for the dispersion coefficient, and the appropriate boundary conditions, is solved by means of a finite element scheme and implemented in the commercial software COMSOL Multiphysics v3.4 (COMSOL 2008).

We confine ourselves to model the steady-state concentration profile of a seeping densegas plume in a two-dimensional vertical section of the lower atmosphere as depicted in Fig. 1. A seeping zone of width $b$ is located at a distance $a$ from the inlet region (left-hand side of the rectangular computational domain). A logarithmic, no-slip surface $\left(\frac{\partial v}{\partial z}=0\right.$, at $z=0$ ) profile for the wind velocity $v$ is imposed at the left of the domain. The value of the height of the computational domain, $H$, was chosen sufficiently large to ensure that the $\mathrm{CO}_{2}$ was exiting from the right-hand vertical boundary. A no-flux boundary condition for the wind velocity was therefore imposed at the top of the computational domain. A more appropriate treatment should include the effects of a stratified atmospheric boundary layer on dense-gas plume dispersion, however we leave this topic for future research. In the near-surface boundary layer we imposed a logarithmic wall function, with a wall offset equal to half the mesh element size (for details of the implementation see, e.g., COMSOL 2008).

The $\mathrm{CO}_{2}$ gas seepage is modelled by imposing a flux boundary condition on the seeping region of width $b$. At the right-hand side of the computational domain we impose a free-flux (Neumann) boundary condition allowing for outflow.

The computational domain is discretised with triangular elements (see Fig.2), and the mesh density is higher near the seepage region to increase numerical accuracy. The elements used for the fluid flow are Lagrange P2-P1 elements (quadratic and linear approximation for the velocity components and pressure, respectively). The concentration is discretised by means of Lagrange-quadratic elements. The number of elements was 7,064, for a total of 75,586 degrees of freedom. For the numerical solution of the fully-coupled system of equations, we used the Pardiso solver (http://www.pardiso-project.org/) as implemented in the COMSOL Multiphysics software. 


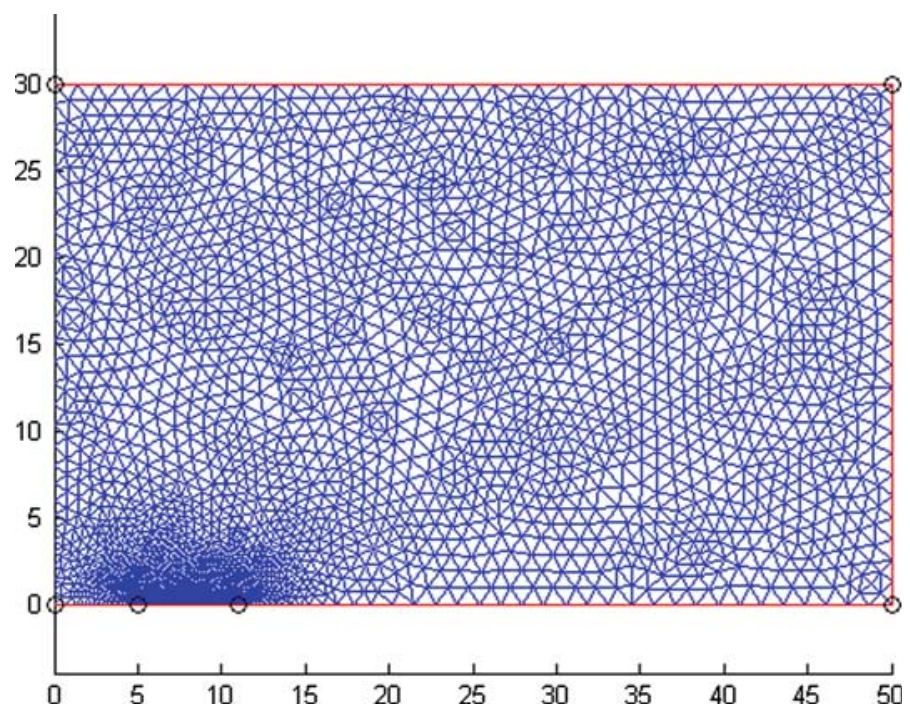

Fig. 2 Typical mesh used for the finite element simulations of density-dependent atmospheric flow and dense-gas transport (units of $(\mathrm{m})$ ). The higher density of the mesh corresponds to the seepage zone

The final set of equations is highly non-linear, and tends to be particularly stiff for low wind speeds and large density contrast. For this reason, we adopted a step-by-step approach to the solution. Given a wind speed, the solution for a higher wind speed was obtained, which was subsequently used as the initial solution for a problem with a lower wind speed. This parametric approach allowed us to achieve convergence for relatively low values of the wind speed. The tolerance for the iterative residual of the system was set equal to $10^{-4}$ to ensure convergence.

We have validated the numerical solutions obtained with our code against an analytical solution valid for passive (no density dependence) transport. The reference analytical solution assumes passive tracer Gaussian dispersion, with a constant wind profile, and can be approximated by the expression (Arya 1999, Eq. 6.45)

$$
c(x, z)=\frac{\int_{-\infty}^{+\infty} c(x=+\infty, z) d z}{\sqrt{4 \pi v(z) D x}} \exp \left(-\frac{v z^{2}}{4 D x}\right),
$$

which is valid at a sufficiently large distance from the injection point. We considered the case of constant velocity $v=1 \mathrm{~m} \mathrm{~s}^{-1}$ and dispersion coefficient $D=2 \mathrm{~m} \mathrm{~s}^{-2}$. The comparison is presented in Fig. 3. The agreement between numerical and analytical solutions is satisfactory at a sufficiently large distance from the injection point, as expected from the assumptions of the analytical solution (8). The difference between the two concentration profiles can be ascribed to the different boundary condition for the seepage: imposed flux in our model versus imposed concentration in (8).

\section{Results}

The relative importance of the density effects on plume dispersion can be characterised by the so-called global Richardson number, 

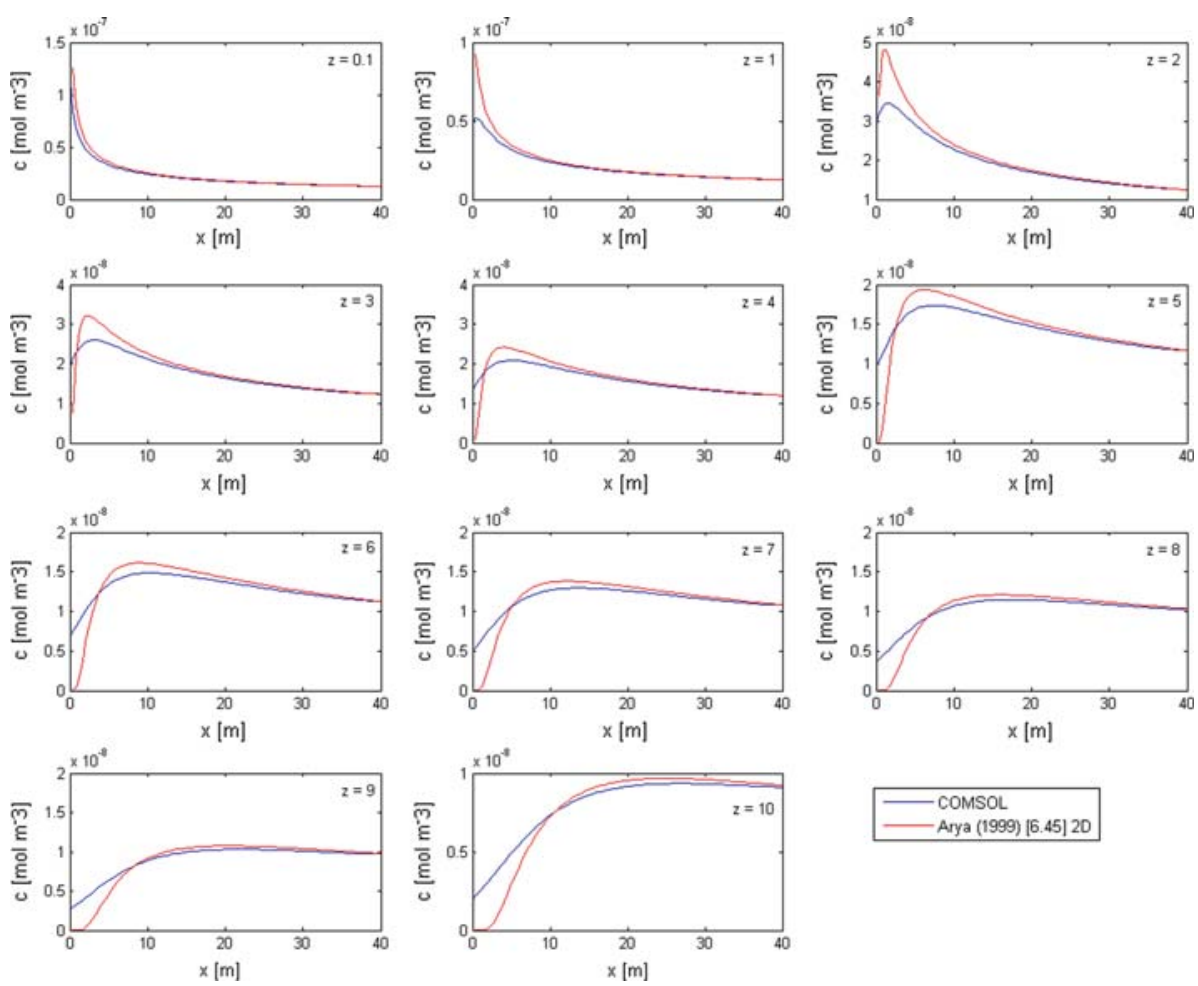

Fig. 3 Validation of the numerical model for the case of a non-dense tracer gas. In blue the solution of our numerical model, and in red the ADE solution in (8). The difference between the two concentration profiles can be ascribed to the different boundary condition for the seepage: imposed flux in our model versus imposed concentration in the ADE model

$$
R i^{\star}=\frac{g_{0}^{\prime} q_{0}}{b U^{3}}
$$

where $q_{0}$ is the volumetric seepage flow rate $\left(\mathrm{m}^{3} \mathrm{~s}^{-1}\right), b$ is the seepage width, $U$ is the wind velocity at the reference altitude $z=10 \mathrm{~m}$, and $g_{0}^{\prime}=g \frac{\rho_{c}-\rho_{a}}{\rho_{a}}$, is the reduced gravity. According to Britter and McQuaid (1988), the transition between passive and density-driven transport occurs at $\left(R i^{\star}\right)^{\frac{1}{3}}=R i<0.15$.

We have performed a series of numerical simulations corresponding to different values of the seepage width $b$, the wind speed $v$, and $\mathrm{CO}_{2}$ seepage flux, $\Phi\left(\mathrm{molm}^{-2} \mathrm{~s}^{-1}\right)$. The size of the two-dimensional (2D) computational domain for all cases is $L=50 \mathrm{~m}, H=30 \mathrm{~m}$.

Figure 4 illustrates this transition threshold graphically for the two limiting values of the seepage length used in this study, i.e., $b=1$ and $b=6$. Our computations cover both the active and passive cases. The reduced Richardson number corresponding to our numerical computations ranges from very low values to a maximum of $R i \simeq 2$.

Figure 5 illustrates a typical simulation for a flux $\Phi=32 \mu \mathrm{mol} \mathrm{m}^{-2} \mathrm{~s}^{-1}$, values of $a=$ $5 \mathrm{~m}, b=2 \mathrm{~m}$, and wind speed at $z=3 \mathrm{~m}$ equal to $0.5 \mathrm{~m} \mathrm{~s}^{-1}$. Only a $20 \times 10$ inset of the $520 \times 30$ computational domain is shown. In this case, the value of the $\mathrm{CO}_{2}$ concentration is smaller than 1 ppmv, a value too low to significantly modify the values of density and 


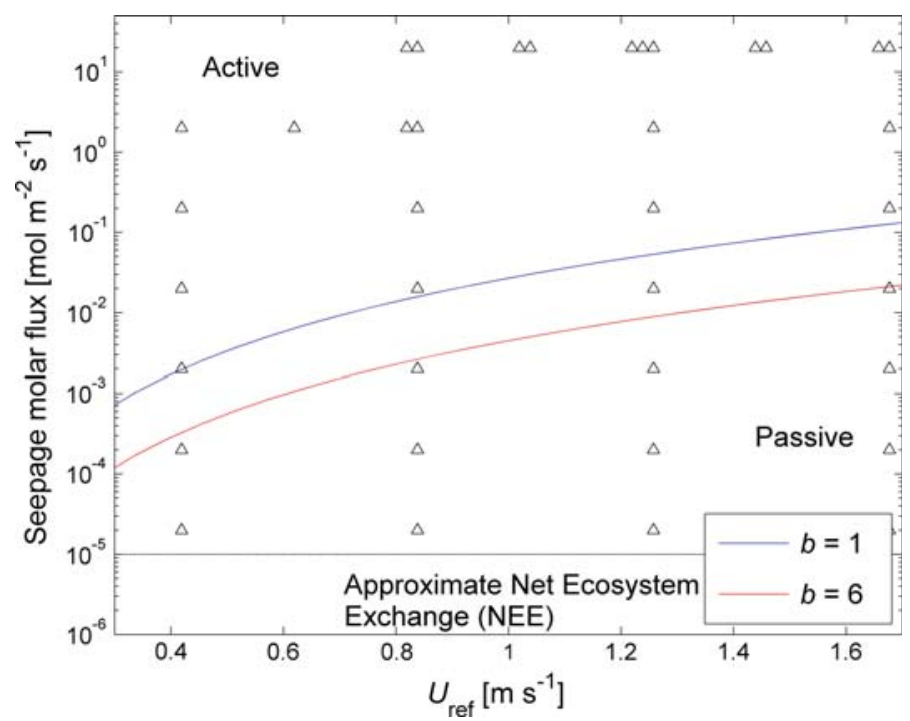

Fig. 4 Summary of the reference velocity values $U_{\text {ref }}$ (estimated at the edge of the seepage area and at an elevation $z=10 \mathrm{~m}$ ) and seepage fluxes for the range of situations explored in this work. The black dashed line represents an approximate estimate of the net ecosystem exchange (NEE). Triangles represent the configurations investigated in this study

viscosity of the air. This can be seen by looking at the flow streamlines that are evidently parallel to the ground surface (unperturbed).

Figure 6 illustrates the effect of the increase in the value of the $\mathrm{CO}_{2}$ flux, which is now $10^{6}$ times larger. The maximum value of the concentration is now on the order of $4 \times 10^{5} \mathrm{ppmv}$, a value sufficiently large to significantly modify the values of density and viscosity of the air. This effect can now be appreciated by looking at the streamlines, which now bend in the region around the seepage area. A significant recirculation area can be identified over the seepage area: the streamlines recirculate the $\mathrm{CO}_{2}$, increasing the concentration build-up and enhancing the upwind tail of the concentration profile. This recirculation process seems not to have received significant attention in the scientific literature.

For intermediate values of the seepage flux between the two extreme cases presented in Figs. 5 and 6, we can find a transition region in which the streamlines are perturbed over the seepage area, but no recirculation region is present; this transition will be analysed in more detail later.

Figure 7 summarises the concentration profiles for the case $b=6 \mathrm{~m}$ and $a=5 \mathrm{~m}$. The distance $x$ is measured from the downwind edge of the seepage area, and the concentration $c(x)$ at ground level $(z=0)$ is expressed in units of $\mathrm{mol} \mathrm{m}^{-3}$. The flux $\Phi$ ranges between $2 \times 10^{-5}$ and $1.8 \times 10^{1} \mu \mathrm{mol} \mathrm{m}{ }^{-2} \mathrm{~s}^{-1}$, and four different wind velocities at $z=3 \mathrm{~m}$ have been considered, namely $v=0.42,0.84,1.26$, and $1.68 \mathrm{~m} \mathrm{~s}^{-1}$, respectively.

The results presented in Fig. 7 suggest the introduction of a non-dimensional concentration $\xi=v c / \Phi$. Figure 8 plots the non-dimensional ground-level concentration profiles. Most of the profiles collapse now on to a single universal curve, with some outliers observed for relatively high values of the $\mathrm{CO}_{2}$ flux, and for lower values of the wind speed. This suggests the existence of a universal scaling for the nondimensional concentration. Figure 9 summarises all the profiles of Fig. 8 (the outliers have been omitted from this plot). 


\section{Concentration, $c$ [ppmv]}
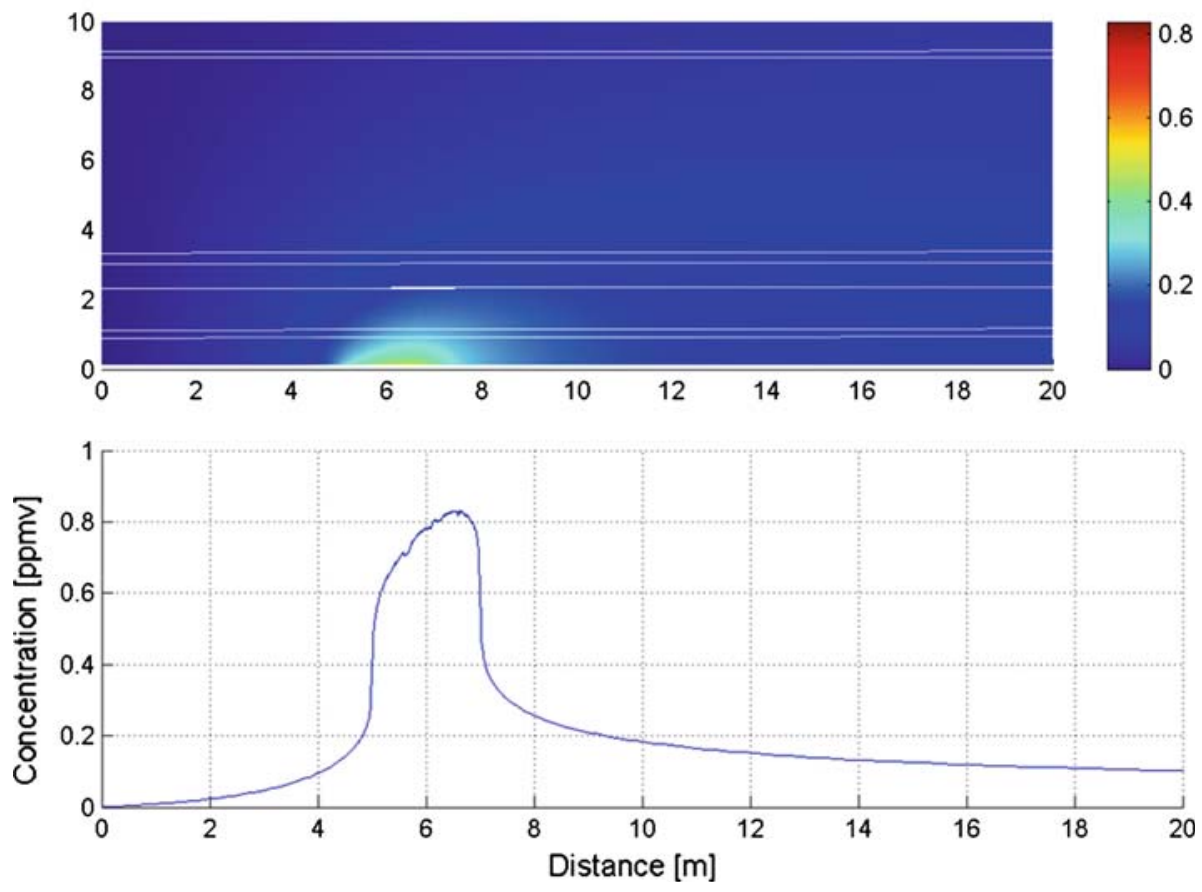

Fig. 5 Concentration map and profile at ground level for a $32 \mu \mathrm{mol} \mathrm{m}^{-2} \mathrm{~s}^{-1} \mathrm{CO}_{2}$ seepage flux

There is clearly a universal scaling of the ground level concentration profiles as a function of the seepage flux and wind velocity, and the solid line in Fig. 9 represents the best-fit relation

$$
\log _{10}(\xi(x))=\sum_{k=0}^{4} \alpha_{k} \log _{10}^{k}(x),
$$

where the coefficients $\alpha_{k}$ of the universal scaling obviously depend on the value of the seepage width $b$. This universal scaling persists also as a function of the elevation $z$ at which the concentration is measured up to $z \simeq 5 \mathrm{~m}$ and then it starts to bifurcate into two stable regimes. This behaviour is illustrated in Fig. 10, where the colour of each curve represents the value of the Richardson number $R i$ of each profile. We find that the threshold between passive and density-dominated regimes appears around the value $R i \simeq 0.5$, a significantly larger value than the 0.15 suggested by Britter and McQuaid (1988). We attribute this discrepancy to our choice of relaxing the Boussinesq assumption, i.e., to our exact accounting of density effects in the inertial terms of the Navier-Stokes equations. The scaling proposed in (10) is consistent with the wind-tunnel near-ground concentration profile measurements presented in Snyder (2001).

In Fig. 11, we classify the same numerical results presented in Fig. 10 according to a different scheme. While the Richardson number is an a priori analysis of the influence of density on dispersion, Fig. 11 presents an a posteriori analysis based on the observation of recirculation zones near the seepage area. The concentration profiles in red are those that exhibit recirculation, i.e., closed-loop streamlines. It can be seen that the transition to 

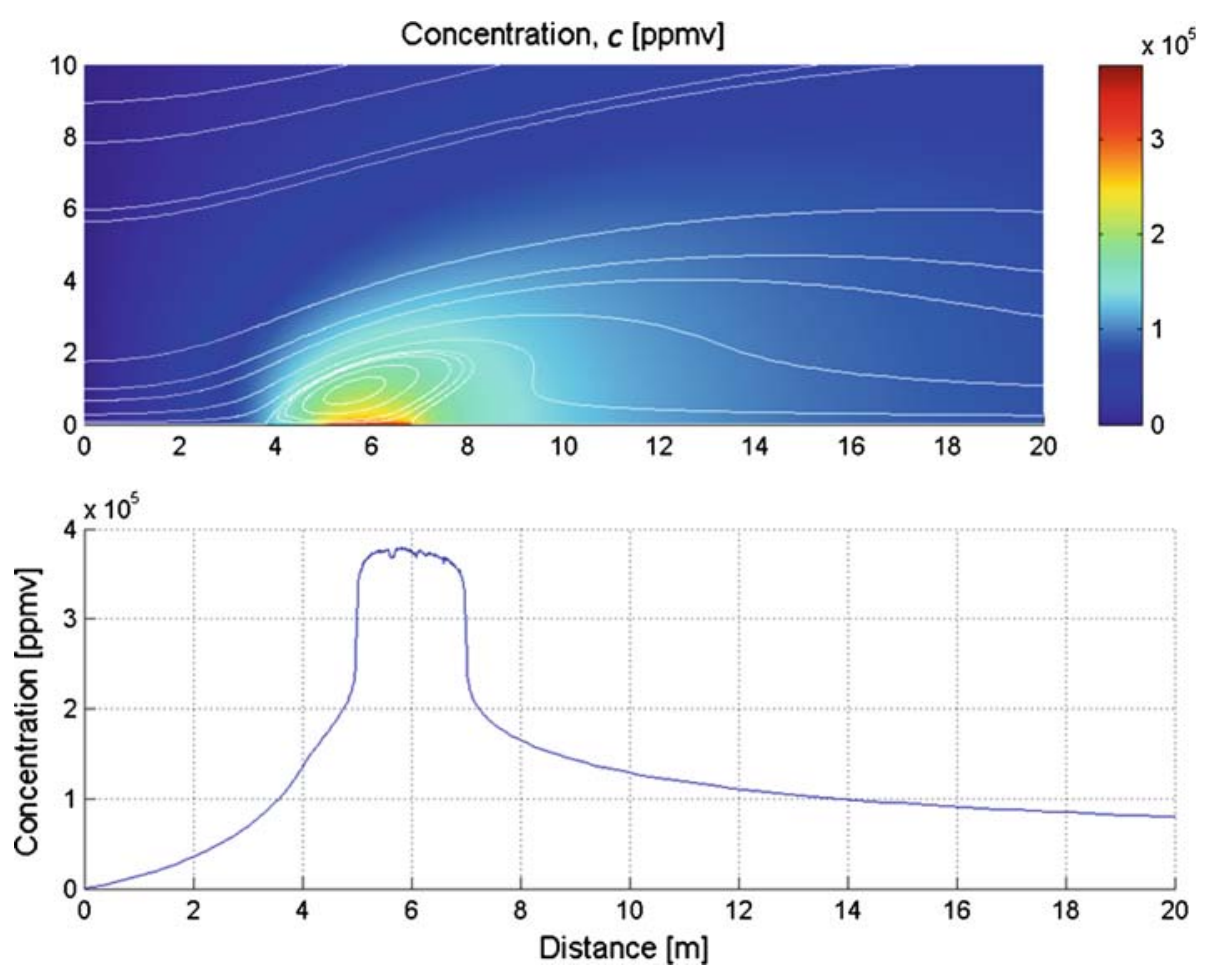

Fig. 6 Concentration map and profile at ground level for a $32 \mathrm{~mol} \mathrm{~m}^{-2} \mathrm{~s}^{-1} \mathrm{CO}_{2}$ seepage flux
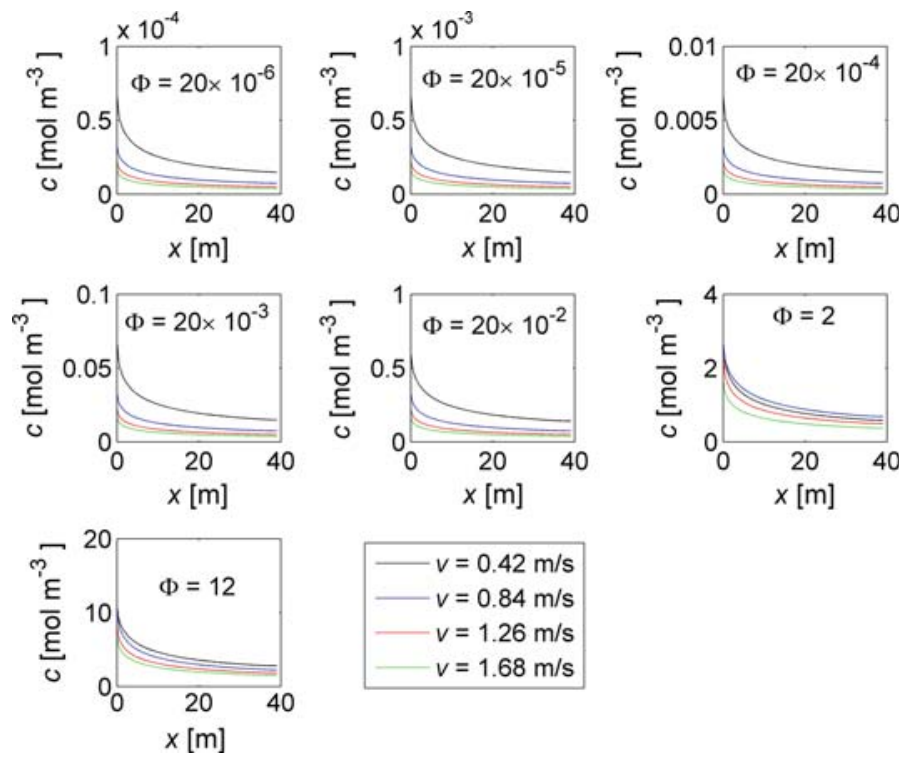

Fig. 7 Concentration profiles at ground level for different level of the seepage flux $\Phi \mu \mathrm{mol} \mathrm{m}^{-2} \mathrm{~s}^{-1}$ and for different wind velocities. The black, blue, red, and green curves correspond to assigned wind velocities at $z=3 \mathrm{~m}$ equal to $v=0.42,0.84,1.26,1.68 \mathrm{~m} \mathrm{~s}^{-1}$, respectively. The seepage width $b=6 \mathrm{~m}$ 

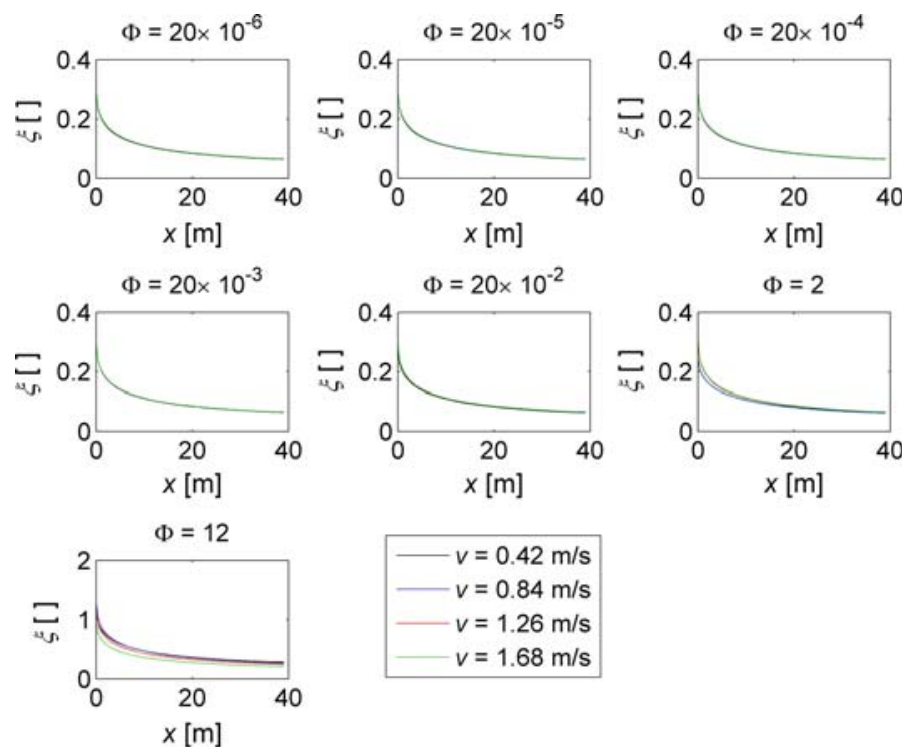

Fig. 8 Normalized concentration profiles at ground level for the case $b=6 \mathrm{~m}$. Deviations from a universal scaling can be observed for relatively high values of the $\mathrm{CO}_{2}$ flux, and for lower values of the wind speed

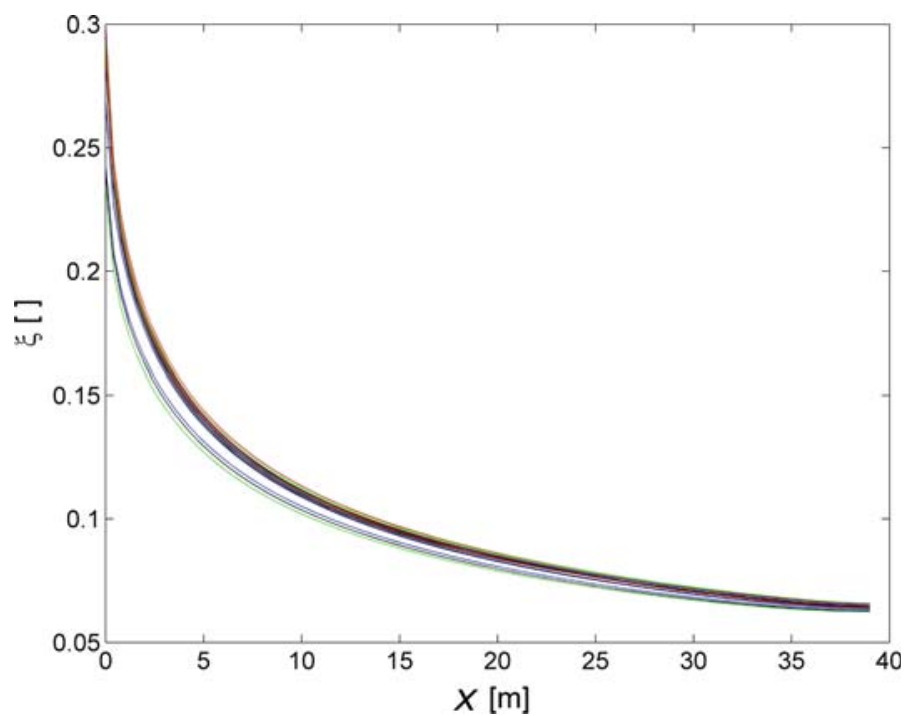

Fig. 9 Universal scaling of the concentration profiles at ground level for different levels of the seepage flux and for different wind velocity for the case of a seepage area that extends linearly for $b=6 \mathrm{~m}$

recirculation-dominated flow happens around $R i \simeq 1$, meaning that we can identify an interval of Richardson numbers $R i \simeq[0.7-1.0]$ that characterises the transition from purely passive to recirculation-dominated, in which density effects are present and manifest themselves by the bulging of the otherwise parallel wind streamlines. The number of configurations in the density-driven regime are, however, clearly insufficient to make any significant 

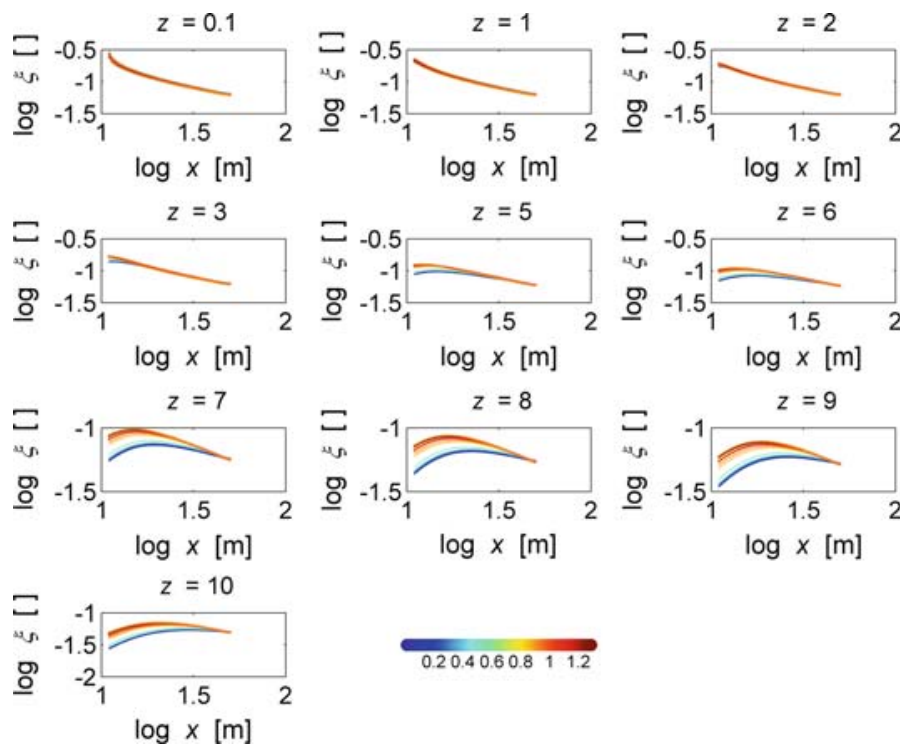

0.20 .40 .60 .811 .2

Fig. 10 Normalized concentration profile at various elevations $z$, for the case $b=6$. Colours indicate the value of the cubic root of the Richardson number. According to Britter and McQuaid (1988), values larger than 0.15 should indicate density effects on transport. Our numerical simulations seem to indicate a threshold around $R i \simeq 0.5$
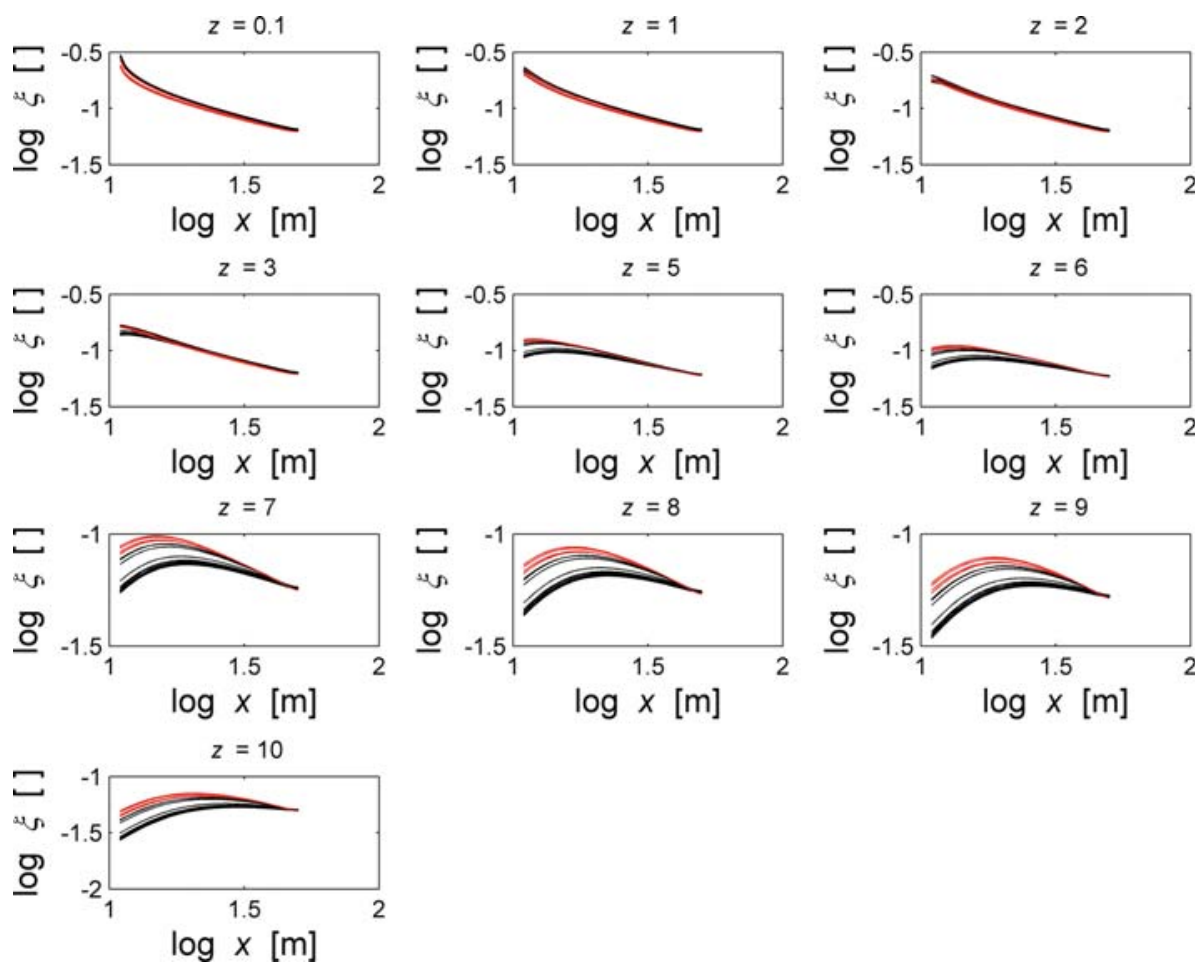

Fig. 11 Same as Fig. 10. In red, the concentration profiles that show recirculation 

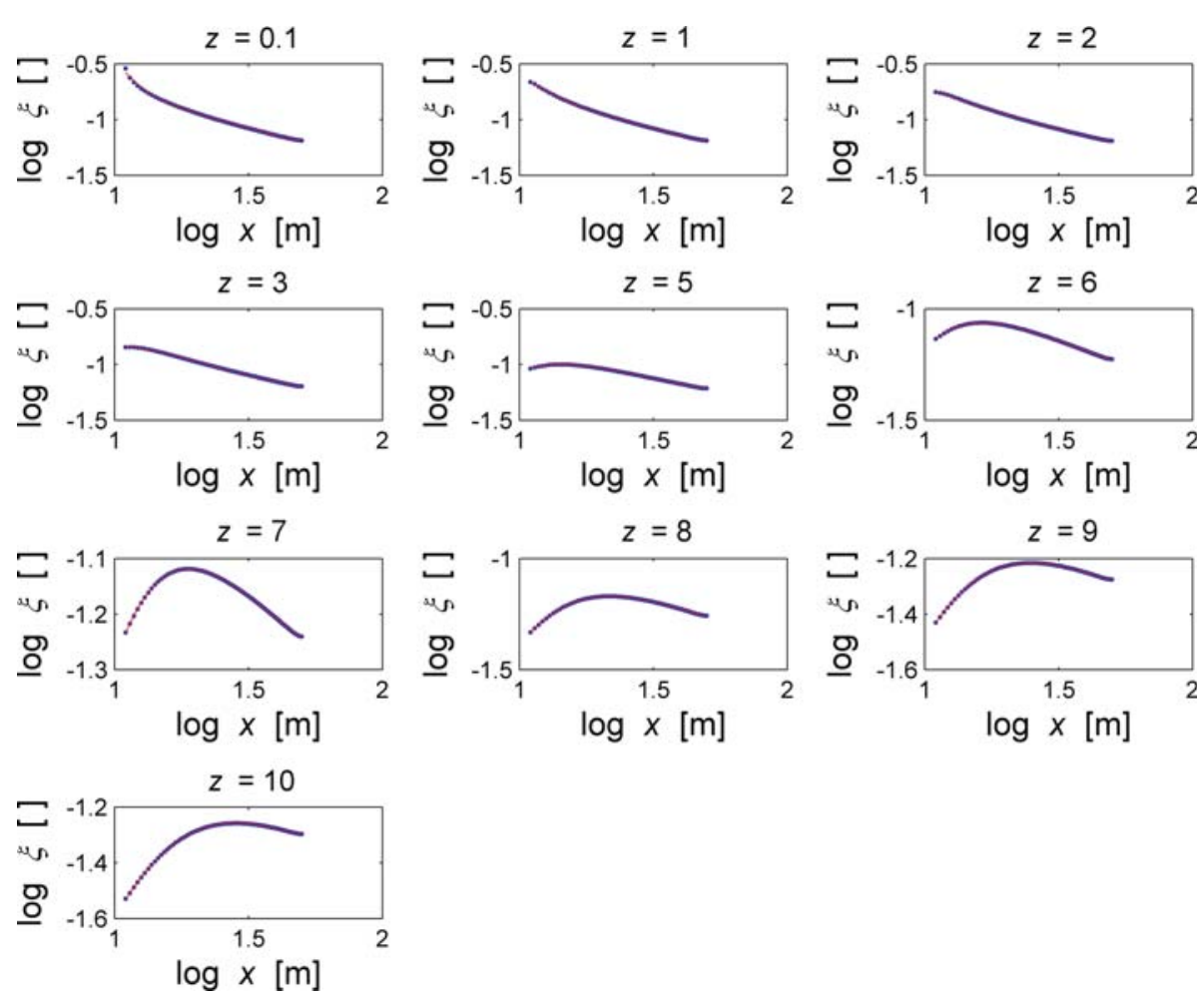

Fig. 12 Same as Fig. 11. The blue dots represent the average value of the concentration profiles in Fig. 11, excluding those that manifest recirculation

statistics and this observation needs further exploration before any general conclusion can be drawn.

Finally, Fig. 12 shows the average concentration profiles at different elevations $z$, for the case $b=6 \mathrm{~m}$ where the recirculating zone cases have been eliminated. Similar figures have been obtained for the cases $b=1,2,3,4$, and $5 \mathrm{~m}$. From the profiles, we can derive now the expressions for the coefficients $\alpha_{k}$, and we can interpolate the coefficients as a function of $z$, by means of fifth-order degree polynomials,

$$
\alpha_{k}(z)=\sum_{j=0}^{5} \beta_{j k} z^{j}
$$

as illustrated in Fig. 13. The interpolating coefficients $\beta_{j k}$ are given in Table 1 as a function of the seepage width $b$. For intermediate values of the seepage width $b$, we suggest a simple linear interpolation of the coefficients.

By means of the coefficients tabulated in Table 1, we are now in the position of being able to estimate the value of the $\mathrm{CO}_{2}$ seepage flux $\Phi$, given a series of $\mathrm{CO}_{2}$ concentration measurements at various elevations $z$, a measured value of the wind velocity $v$, and an estimated seepage width $b$, without resorting to laborious numerical solutions. This means in principle that a technician in the field can give an estimate of the seepage flux with operations that can be easily implemented on a pocket calculator. For example, consider the case of a large-scale GCS site at which monitoring and detection methods (see, e.g., Oldenburg et al. 


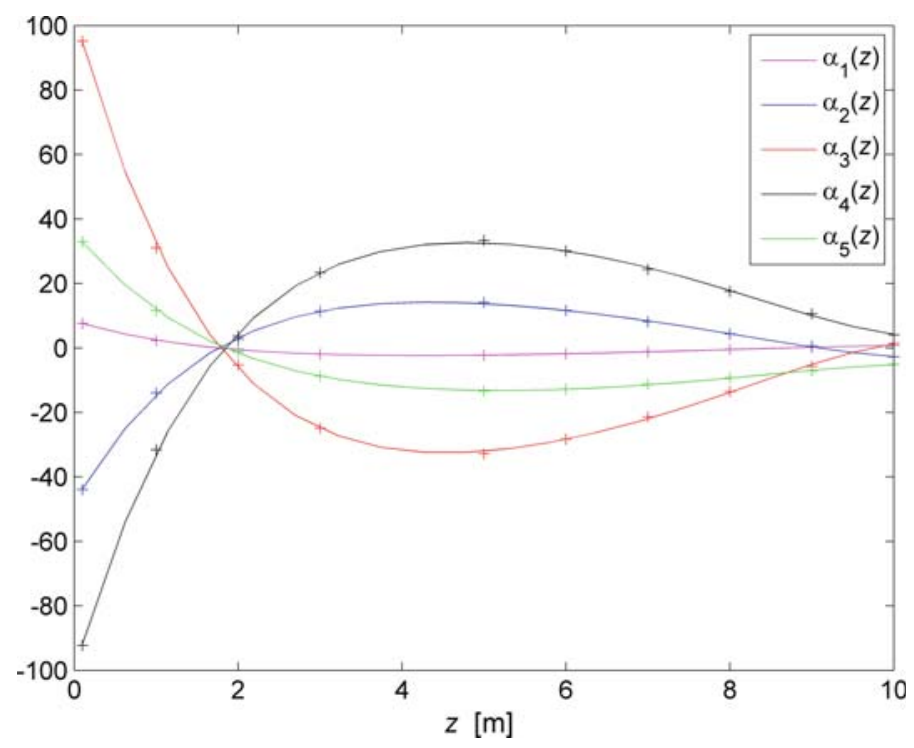

Fig. 13 The coefficients $\alpha_{k}$ (indicated by points with a + sign) represent the coefficients of the fourth-order polynomial interpolation between the log of the horizontal distance from the source and the $\log$ of the normalized concentration for the case $b=6$. The coloured solid lines represent the fifth-order polynomials interpolating the coefficients $\alpha_{k}(z)$ as a function of the elevation $z$, in the range from 1 to $10 \mathrm{~m}$. In Table 1 , we summarize the coefficients of this interpolation for different values of the seepage length $b$

2003; Lewicki etal. 2007; Leuning etal. 2008) had identified the presence of anomalous $\mathrm{CO}_{2}$ in an area, and more detailed investigation (see, e.g., Cortis et al. 2008) had pinpointed an elongated seepage area (e.g., along a fault trace, or $\operatorname{crack}(\mathrm{s})$ in a paved surface). A field technician could carry out an inexpensive survey over the course of an afternoon using, (i) an anemometer set up when the flow is generally transverse to the fault, and (ii) a hand-held $\mathrm{CO}_{2}$ infrared (IR) detector to measure $\mathrm{CO}_{2}$ concentration at ground level along $10-50 \mathrm{~m}$ transects over the suspected seepage area. Repeated transects in a grid pattern that would presumably produce a peak concentration near the seepage area, rapid decline in concentration upwind of the seepage area (allowing estimation of the seepage area width, $b$ ), and more gentle decline in concentration downwind of the seepage area, consistent with dilution due to turbulent mixing and dispersion. Without recourse to any numerical computation, the concentration and wind velocity data could be suitably averaged and combined with the $\alpha_{k}$ parameters from Table 1 to solve for the estimate flux $\Phi$.

More complex estimation schemes can be devised, which involve the solution of overdetermined linear systems of equations, should a sufficiently large number of measurements at different elevations $z$, and distances $x$ be available. It is important to note here that the implicit assumptions used to calculate the concentration profiles (e.g., 2D vs. 3D profiles, multiplier of the dispersion coefficient $f$, topographic conditions), may be relaxed and new interpolation functions be deduced from the corresponding numerical computations.

\section{Conclusions}

We have presented a numerical study aimed at understanding the effects of density on the transport behaviour of $\mathrm{CO}_{2}$ plumes seeping out of the ground. The model is aimed at 
Table 1 Table of interpolation coefficients for the normalised concentration as a function of the elevation $z$ $(\mathrm{m})$, and the seepage length $b(\mathrm{~m})$

\begin{tabular}{|c|c|c|c|c|c|c|}
\hline & $z^{5}$ & $z^{4}$ & $z^{3}$ & $z^{2}$ & $z^{1}$ & $z^{0}$ \\
\hline \multicolumn{7}{|l|}{$b=1$} \\
\hline$\alpha_{1}$ & $-2.51 \times 10^{-5}$ & $9.53 \times 10^{-4}$ & $-1.46 \times 10^{-2}$ & $1.09 \times 10^{-1}$ & $-3.55 \times 10^{-1}$ & $2.84 \times 10^{-1}$ \\
\hline$\alpha_{2}$ & $2.83 \times 10^{-4}$ & $-1.09 \times 10^{-2}$ & $1.70 \times 10^{-1}$ & -1.30 & 4.35 & -3.55 \\
\hline$\alpha_{3}$ & $-1.16 \times 10^{-3}$ & $4.57 \times 10^{-2}$ & $-7.24 \times 10^{-1}$ & 5.67 & $-1.97 \times 10^{1}$ & $1.66 \times 10^{1}$ \\
\hline$\alpha_{4}$ & $2.03 \times 10^{-3}$ & $-8.19 \times 10^{-2}$ & 1.33 & $-1.08 \times 10^{1}$ & $3.91 \times 10^{1}$ & $-3.48 \times 10^{1}$ \\
\hline$\alpha_{5}$ & $-1.27 \times 10^{-3}$ & $5.28 \times 10^{-2}$ & $-8.87 \times 10^{-1}$ & 7.41 & $-2.86 \times 10^{1}$ & $2.57 \times 10^{1}$ \\
\hline \multicolumn{7}{|l|}{$b=2$} \\
\hline$\alpha_{1}$ & $-4.36 \times 10^{-6}$ & $3.26 \times 10^{-4}$ & $-7.65 \times 10^{-3}$ & $7.73 \times 10^{-2}$ & $-3.13 \times 10^{-1}$ & $2.96 \times 10^{-1}$ \\
\hline$\alpha_{2}$ & $3.48 \times 10^{-5}$ & $-3.42 \times 10^{-3}$ & $8.76 \times 10^{-2}$ & $-9.28 \times 10^{-1}$ & 3.90 & -3.77 \\
\hline$\alpha_{3}$ & $-6.23 \times 10^{-5}$ & $1.25 \times 10^{-2}$ & $-3.64 \times 10^{-1}$ & 4.09 & $-1.80 \times 10^{1}$ & $1.80 \times 10^{1}$ \\
\hline$\alpha_{4}$ & $-9.14 \times 10^{-5}$ & $-1.81 \times 10^{-2}$ & $6.45 \times 10^{-1}$ & -7.82 & $3.65 \times 10^{1}$ & $-3.85 \times 10^{1}$ \\
\hline $\begin{array}{l}\alpha_{5} \\
b=3\end{array}$ & $2.44 \times 10^{-4}$ & $7.52 \times 10^{-3}$ & $-4.04 \times 10^{-1}$ & \multicolumn{2}{|c|}{$b=3$} & $3.00 \times 10^{1}$ \\
\hline$\alpha_{1}$ & $-2.86 \times 10^{-6}$ & $2.56 \times 10^{-4}$ & $-6.82 \times 10^{-3}$ & $7.81 \times 10^{-2}$ & $-3.60 \times 10^{-1}$ & $4.05 \times 10^{-1}$ \\
\hline$\alpha_{2}$ & $2.11 \times 10^{-5}$ & $-2.74 \times 10^{-3}$ & $8.05 \times 10^{-2}$ & $-9.68 \times 10^{-1}$ & 4.62 & -5.29 \\
\hline$\alpha_{3}$ & $-2.25 \times 10^{-5}$ & $1.03 \times 10^{-2}$ & $-3.47 \times 10^{-1}$ & 4.43 & $-2.20 \times 10^{1}$ & $2.59 \times 10^{1}$ \\
\hline$\alpha_{4}$ & $-1.22 \times 10^{-4}$ & $-1.55 \times 10^{-2}$ & $6.44 \times 10^{-1}$ & -8.83 & $4.64 \times 10^{1}$ & $-5.70 \times 10^{1}$ \\
\hline$\alpha_{5}$ & $2.29 \times 10^{-4}$ & $7.05 \times 10^{-3}$ & $-4.30 \times 10^{-1}$ & 6.45 & $-3.63 \times 10^{1}$ & $4.67 \times 10^{1}$ \\
\hline \multicolumn{7}{|l|}{$b=4$} \\
\hline$\alpha_{1}$ & $-2.86 \times 10^{-6}$ & $2.56 \times 10^{-4}$ & $-6.82 \times 10^{-3}$ & $7.81 \times 10^{-2}$ & $-3.60 \times 10^{-1}$ & $4.05 \times 10^{-1}$ \\
\hline$\alpha_{2}$ & $2.11 \times 10^{-5}$ & $-2.74 \times 10^{-3}$ & $8.05 \times 10^{-2}$ & $-9.68 \times 10^{-1}$ & 4.62 & -5.29 \\
\hline$\alpha_{3}$ & $-2.25 \times 10^{-5}$ & $1.03 \times 10^{-2}$ & $-3.47 \times 10^{-1}$ & 4.43 & $-2.20 \times 10^{1}$ & $2.59 \times 10^{1}$ \\
\hline$\alpha_{4}$ & $-1.22 \times 10^{-4}$ & $-1.55 \times 10^{-2}$ & $6.44 \times 10^{-1}$ & -8.83 & $4.64 \times 10^{1}$ & $-5.70 \times 10^{1}$ \\
\hline$\alpha_{5}$ & $2.29 \times 10^{-4}$ & $7.05 \times 10^{-3}$ & $-4.30 \times 10^{-1}$ & 6.45 & $-3.63 \times 10^{1}$ & $4.67 \times 10^{1}$ \\
\hline \multicolumn{7}{|l|}{$b=5$} \\
\hline$\alpha_{1}$ & $-6.25 \times 10^{-6}$ & $3.66 \times 10^{-4}$ & $-8.38 \times 10^{-3}$ & $9.08 \times 10^{-2}$ & $-4.16 \times 10^{-1}$ & $4.85 \times 10^{-1}$ \\
\hline$\alpha_{2}$ & $6.54 \times 10^{-5}$ & $-4.20 \times 10^{-3}$ & $1.02 \times 10^{-1}$ & -1.14 & 5.40 & -6.40 \\
\hline$\alpha_{3}$ & $-2.38 \times 10^{-4}$ & $1.75 \times 10^{-2}$ & $-4.53 \times 10^{-1}$ & 5.33 & $-2.62 \times 10^{1}$ & $3.17 \times 10^{1}$ \\
\hline$\alpha_{4}$ & $3.38 \times 10^{-4}$ & $-3.11 \times 10^{-2}$ & $8.79 \times 10^{-1}$ & $-1.09 \times 10^{1}$ & $5.59 \times 10^{1}$ & $-7.03 \times 10^{1}$ \\
\hline $\begin{array}{l}\alpha_{5} \\
b=6\end{array}$ & $-1.34 \times 10^{-4}$ & $1.96 \times 10^{-2}$ & $-6.22 \times 10^{-1}$ & 8.17 & $-4.44 \times 10^{1}$ & $5.85 \times 10^{1}$ \\
\hline$\alpha_{1}$ & $-3.04 \times 10^{-5}$ & $1.07 \times 10^{-3}$ & $-1.56 \times 10^{-2}$ & $1.25 \times 10^{-1}$ & $-5.05 \times 10^{-1}$ & $5.93 \times 10^{-1}$ \\
\hline$\alpha_{2}$ & $3.66 \times 10^{-4}$ & $-1.32 \times 10^{-2}$ & $1.98 \times 10^{-1}$ & -1.61 & 6.66 & -7.91 \\
\hline$\alpha_{3}$ & $-1.59 \times 10^{-3}$ & $5.98 \times 10^{-2}$ & $-9.25 \times 10^{-1}$ & 7.72 & $-3.28 \times 10^{1}$ & $3.95 \times 10^{1}$ \\
\hline$\alpha_{4}$ & $2.92 \times 10^{-3}$ & $-1.17 \times 10^{-1}$ & 1.89 & $-1.67 \times 10^{1}$ & $7.12 \times 10^{1}$ & $-8.85 \times 10^{1}$ \\
\hline$\alpha_{5}$ & $-1.80 \times 10^{-3}$ & $8.06 \times 10^{-2}$ & -1.41 & $1.27 \times 10^{1}$ & $-5.77 \times 10^{1}$ & $7.45 \times 10^{1}$ \\
\hline
\end{tabular}


improving the confidence in seepage flux field measurement values, a crucial step in providing better constraints for the optimization of monitoring networks, and thereby minimizing their costs. We simulated small-scale atmospheric flows by means of a $k-\varepsilon$ turbulence model of the NS equations, where the density and viscosity depend on the local $\mathrm{CO}_{2}$ concentration value. We have identified a transition region for the Richardson number that leads to a density-dominated regime, and illustrated its effects on the contaminant transport. By defining a non-dimensional concentration normalised with respect to the value of wind velocity and seepage flux, we have identified a universal scaling for the concentration profiles, which breaks down only at extremely high values of the flux, or low values of the wind speed. The combination of such large $\mathrm{CO}_{2}$ fluxes and no-wind conditions that lead to recirculation zones, i.e., closed loop streamlines of the wind velocity field, while extremely important from a safety and risk-assessment perspective, is unlikely to be found in the ordinary operation of a monitoring network, and urgent responses are needed instead (Cortis et al. 2008). Based on our numerical simulations, we provided simple interpolation formulae that can be used in the field to estimate seepage fluxes, given other atmospheric concentration measurements, and the measurement of the wind velocity. Numerical simulations aimed at relaxing the initial model assumptions used in this work will make it possible to extend this simple interpolation scheme and generalise it to less idealised field situations.

Acknowledgements This work was carried out in the ZERT project funded by the Assistant Secretary for Fossil Energy, Office of Sequestration, Hydrogen, and Clean Coal Fuels, through the National Energy Technology Laboratory, U.S. Department of Energy under Contract No. DE-AC02-05CH11231.

Open Access This article is distributed under the terms of the Creative Commons Attribution Noncommercial License which permits any noncommercial use, distribution, and reproduction in any medium, provided the original author(s) and source are credited.

\section{References}

Arya S (1999) Air pollution meteorology and dispersion. Oxford University Press, New York, 420 pp

Benson S (2006) Monitoring carbon dioxide sequestration in deep geological formations for inventory verification and carbon credit. In: SPE-102833, 2006 annual technical conference and exhibition, Society of Petroleum Engineers, San Antonio, TX, 24-27 September

Berkowitz B, Cortis A, Dentz M, Scher H (2006) modeling non-fickian transport in geological formations as a continuous time random walk. Rev Geophys 44:1-49

Britter RE, McQuaid J (1988) Workbook on the dispersion of dense gases. Contract Research Report 17/1988. Health and Safety Executive, Sheffield, UK

COMSOL (2008) Multiphysics user's guide. COMSOL AB, v3.4 edn

Cortis A, Oldenburg CM, Benson SM (2008) The role of optimality in characterizing $\mathrm{CO}_{2}$ seepage from geologic carbon sequestration sites. Int J Greenh Gas Control 2(4):640-652

Costa A, Macedonio G, Chiodini G (2005) Numerical model of gas dispersion emitted from volcanic sources. Ann Geophys Italy 48(4-5):805-815

Costa A, Chiodini G, Granieri D, Folch A, Hankin RKS, Caliro S, Avino R, Cardellini C (2008) A shallowlayer model for heavy gas dispersion from natural sources: application and hazard assessment at Caldara di Manziana, Italy. Geochem Geophys Geosystems 9:Q03002. doi:10.1029/2007GC001762

Davis P, Reimer A, Sakiyama S, Slawson P (1986) Short-range atmospheric dispersion over a heterogeneous surface-i. Lateral dispersion. Atmos Environ 20(1):41-50. doi:10.1016/0004-6981(86)90205-2

Hanna S, Chang J (2001) Use of the kit fox field data to analyze dense gas dispersion modeling issues. Atmos Environ 35:2231-2242

Hanna S, Steinberg K (2001) Overview of petroleum research forum (perf) dense gas dispersion modeling project. Atmos Environ 35:2223-2229

Hanna S, Dharmavaram S, Zhang J, Sykes I, Witlox H, Khajehnajafi S, Koslan K (2008) Comparison of six widely-used dense gas dispersion models for three recent chlorine railcar accidents. Process Saf Prog 27(3):248-259. doi:10.1002/prs.10257 
INEEL (1999) Supplemental response to the type a accident investigation board report of the July 28, 1998, fatality and multiple injuries resulting from release of carbon dioxide at building 648, test reactor area, idaho national engineering and environmental laboratory. Technical report INEEL/EXT-99-00282. Idaho National Engineering and Environmental Laboratory

Leuning R, Etheridge D, Luhar A, Dunse B (2008) Atmospheric monitoring and verification technologies for $\mathrm{CO}_{2}$ geosequestration. Int J Greenh Gas Control 2(3):401-414. doi:10.1016/j.ijggc.2008.01.002, eGU General Assembly 2007: Advances in $\mathrm{CO}_{2}$ storage in geological systems-EGU (2007)

Lewicki J, Oldenburg C, Dobeck L, Spangler L (2007) Surface $\mathrm{CO}_{2}$ leakage during two shallow subsurface $\mathrm{CO}_{2}$ releases. Geophys Res Lett 34:L24402. doi:101029/2007GL032047

Macedonio G, Costa A (2002) Finite element modeling of gas dispersion in the atmosphere. In: Buccianti A, Marini L, Ottonello G, Vasell O (eds) Proceedings of the Arezzo seminar in fluids geochemistry. Pacini Editore, Ospedaletto, pp 147-159

Moridis G, Kowalsky M, Pruess K (2008) Tough+hydrate v1.0 users manual: a code for the simulation of system behavior in hydrate-bearing geologic media. Report LBNL-0149E. Lawrence Berkeley National Laboratory

Oldenburg CM, Unger AJA (2004) Coupled vadose zone and atmospheric surface-layer transport of carbon dioxide from geologic carbon sequestration sites. Vadose Zone J 3:3848-3857

Oldenburg C, Lewicki J, Hepple R (2003) Near-surface monitoring strategies for geologic carbon dioxide storage verification. Report LBNL-54089. Lawrence Berkeley National Laboratory

Pasquill F, Smith FB (1983) Atmospheric diffusion, 3rd edn. Ellis Horwood, Chichester, 438 pp

Peng DY, Robinson DB (1976) A new two-constant equation of state. Ind Eng Chem Fundam 15:59-64

Reagan M (2008) Webgaseos online application v1.0. Report LBNL/PUB-3188. Lawrence Berkeley National Laboratory. http://lnx.lbl.gov/gaseos/

Snyder W (2001) Wind-tunnel study of entrainment in two-dimensional dense-gas plumes at the eps's fluid modeling facility. Atmos Environ 35:2285-2304

Stull R (1988) An introduction to boundary layer meteorology. Kluwer Academic Publishers, Dordrecht, $666 \mathrm{pp}$

Tu J, Yeoh GH, Liu C (2008) Computational fluid dynamics: a practical approach. Butterworth-Heinemann, $472 \mathrm{pp}$

Walas SM (1985) Phase equilibria in chemical engineering. Butterworth-Heinemann, 671 pp 